Publ. Mat. 62 (2018), 615-639

DOI: 10.5565 /PUBLMAT6221808

\title{
DETERMINANTS OF LAPLACIANS ON HILBERT MODULAR SURFACES
}

\author{
YASURo Gon
}

\begin{abstract}
We study regularized determinants of Laplacians acting on the space of Hilbert-Maass forms for the Hilbert modular group of a real quadratic field. We show that these determinants are described by Selberg type zeta functions introduced in $[5,6]$.
\end{abstract}

2010 Mathematics Subject Classification: 11M36, 11F72, 58J52.

Key words: Hilbert modular surface, Selberg zeta function, regularized determinant.

\section{Introduction}

Determinants of the Laplacian $\Delta$ acting on the space of Maass forms on a hyperbolic Riemann surface $X$ are studied by many authors. (See for example $[\mathbf{1 5}, \mathbf{2}, \mathbf{1 1}, \mathbf{1 0}]$.) It is known that the determinants of $\Delta$ are described by the Selberg zeta function (cf. [16]) for $X$.

On the other hand, two Laplacians $\Delta^{(1)}, \Delta^{(2)}$ act on the space of Hilbert-Maass forms on the Hilbert modular surface $X_{K}$ of a real quadratic field $K$. For this reason, it seems that there are no explicit formulas for "Determinants of Laplacians" on $X_{K}$ until now. In this article we consider regularized determinants of the first Laplacian $\Delta^{(1)}$ acting on its certain subspaces $V_{m}^{(2)}$, indexed by $m \in 2 \mathbb{N}$. We show that these determinants are described by Selberg type zeta functions for $X_{K}$ introduced in $[\mathbf{5}, \mathbf{6}]$.

Let $K / \mathbb{Q}$ be a real quadratic field with class number one and $\mathcal{O}_{K}$ be the ring of integers of $K$. Let $D$ be the discriminant of $K$ and $\varepsilon>1$ be the fundamental unit of $K$. We denote the generator of $\operatorname{Gal}(K / \mathbb{Q})$ by $\sigma$ and put $a^{\prime}:=\sigma(a)$ for $a \in K$. We also put $\gamma^{\prime}=\left(\begin{array}{cc}a^{\prime} & b^{\prime} \\ c^{\prime} & d^{\prime}\end{array}\right)$ for $\gamma=\left(\begin{array}{ll}a & b \\ c & d\end{array}\right) \in \operatorname{PSL}\left(2, \mathcal{O}_{K}\right)$. Let $\Gamma_{K}=\left\{\left(\gamma, \gamma^{\prime}\right) \mid \gamma \in \operatorname{PSL}\left(2, \mathcal{O}_{K}\right)\right\}$ be the Hilbert modular group of $K$. It is known that $\Gamma_{K}$ is a co-finite (non-cocompact) irreducible discrete subgroup of $\operatorname{PSL}(2, \mathbb{R}) \times \operatorname{PSL}(2, \mathbb{R})$

This work was partially supported by JSPS Grant-in-Aid for Scientific Research (C) no. 26400017 and no. 17K05178. 
and $\Gamma_{K}$ acts on the product $\mathbb{H}^{2}$ of two copies of the upper half plane $\mathbb{H}$ by component-wise linear fractional transformation. $\Gamma_{K}$ has only one cusp $(\infty, \infty)$, i.e. $\Gamma_{K}$-inequivalent parabolic fixed point. $X_{K}:=\Gamma_{K} \backslash \mathbb{H}^{2}$ is called the Hilbert modular surface.

Let $\left(\gamma, \gamma^{\prime}\right) \in \Gamma_{K}$ be hyperbolic-elliptic, i.e., $|\operatorname{tr}(\gamma)|>2$ and $\left|\operatorname{tr}\left(\gamma^{\prime}\right)\right|<2$. Then the centralizer of hyperbolic-elliptic $\left(\gamma, \gamma^{\prime}\right)$ in $\Gamma_{K}$ is infinite cyclic.

Definition 1.1 (Selberg type zeta function for $\Gamma_{K}$ with the weight $\left.(0, m)\right)$. For an even integer $m \geq 2$, we define

$$
\text { (1.1) } Z_{m}(s):=\prod_{\left(p, p^{\prime}\right) \in \mathrm{P} \Gamma_{\mathrm{HE}}} \prod_{n=0}^{\infty}\left(1-e^{i(m-2) \omega} N(p)^{-(n+s)}\right)^{-1} \text { for } \operatorname{Re}(s)>1 \text {. }
$$

Here, $\left(p, p^{\prime}\right)$ run through the set of primitive hyperbolic-elliptic $\Gamma_{K^{-}}$ conjugacy classes of $\Gamma_{K}$, and $\left(p, p^{\prime}\right)$ is conjugate in $\operatorname{PSL}(2, \mathbb{R})^{2}$ to

$$
\left(p, p^{\prime}\right) \sim\left(\left(\begin{array}{cc}
N(p)^{1 / 2} & 0 \\
0 & N(p)^{-1 / 2}
\end{array}\right),\left(\begin{array}{cc}
\cos \omega & -\sin \omega \\
\sin \omega & \cos \omega
\end{array}\right)\right) .
$$

Here, $N(p)>1, \omega \in(0, \pi)$, and $\omega \notin \pi \mathbb{Q}$. The product is absolutely convergent for $\operatorname{Re}(s)>1$.

Analytic properties of $Z_{m}(s)$ are known.

Theorem 1.2 ([6, Theorems 5.3 and 6.5]). For an even integer $m \geq 2$, $Z_{m}(s)$ a priori defined for $\operatorname{Re}(s)>1$ has a meromorphic extension over the whole complex plane.

In this article, we also consider "the square root of $Z_{2}(s)$ ".

Definition $1.3\left(\sqrt{Z_{2}(s)}\right)$.

$$
\begin{aligned}
\sqrt{Z_{2}(s)} & :=\prod_{\left(p, p^{\prime}\right) \in \mathrm{P} \Gamma_{\mathrm{HE}}} \prod_{n=0}^{\infty}\left(1-N(p)^{-(n+s)}\right)^{-1 / 2} \\
& =\exp \left(\frac{1}{2} \sum_{\left(p, p^{\prime}\right)} \sum_{k=1}^{\infty} \frac{1}{k} \frac{N(p)^{-k s}}{1-N(p)^{-k}}\right) \text { for } \operatorname{Re}(s)>1 .
\end{aligned}
$$

By [6, Theorem 6.5] and the fact that the Euler characteristic of $X_{K}$ is even (see Lemma 2.2), we see that $\frac{d}{d s} \log Z_{2}(s)$ has even integral residues at any poles. Therefore, we find that $\sqrt{Z_{2}(s)}$ has a meromorphic continuation to the whole complex plane.

Let us introduce the completed Selberg type zeta functions $\widehat{Z}_{2}^{\frac{1}{2}}(s)$ and $\widehat{Z}_{m}(s)(m \geq 4)$, which are invariant under $s \rightarrow 1-s$. (See $[6$, Theorems 5.4 and 6.6].) 
Definition 1.4 (Completed Selberg zeta functions).

$$
\widehat{Z}_{2}^{\frac{1}{2}}(s):=\sqrt{Z_{2}(s)} Z_{\mathrm{id}}^{\frac{1}{2}}(s) Z_{\mathrm{ell}}^{\frac{1}{2}}(s ; 2) Z_{\mathrm{par} / \mathrm{sct}}^{\frac{1}{2}}(s ; 2) Z_{\mathrm{hyp} 2 / \mathrm{sct}}^{\frac{1}{2}}(s ; 2)
$$

with

$$
\begin{gathered}
Z_{\mathrm{id}}^{\frac{1}{2}}(s):=\left(\Gamma_{2}(s) \Gamma_{2}(s+1)\right)^{\zeta_{K}(-1)}, \quad Z_{\mathrm{ell}}^{\frac{1}{2}}(s ; 2):=\prod_{j=1}^{N} \prod_{l=0}^{\nu_{j}-1} \Gamma\left(\frac{s+l}{\nu_{j}}\right)^{\frac{\nu_{j}-1-2 l}{2 \nu_{j}}}, \\
Z_{\mathrm{par} / \mathrm{sct}}^{\frac{1}{2}}(s ; 2):=\varepsilon^{-s}, \quad Z_{\mathrm{hyp} 2 / \mathrm{sct}}^{\frac{1}{2}}(s ; 2):=\zeta_{\varepsilon}(s) . \\
(1.4) \quad \widehat{Z}_{m}(s):=Z_{m}(s) Z_{\mathrm{id}}(s) Z_{\mathrm{ell}}(s ; m) Z_{\mathrm{hyp} 2 / \mathrm{sct}}(s ; m) \quad(m \geq 4)
\end{gathered}
$$

with

$$
\begin{gathered}
Z_{\mathrm{id}}(s):=\left(\Gamma_{2}(s) \Gamma_{2}(s+1)\right)^{2 \zeta_{K}(-1)}, \\
Z_{\mathrm{ell}}(s ; m):=\prod_{j=1}^{N} \prod_{l=0}^{\nu_{j}-1} \Gamma\left(\frac{s+l}{\nu_{j}}\right)^{\frac{\nu_{j}-1-\alpha_{l}(m, j)-\overline{\alpha_{l}}(m, j)}{\nu_{j}}}, \\
Z_{\mathrm{hyp} 2 / \mathrm{sct}}(s ; m):=\zeta_{\varepsilon}\left(s+\frac{m}{2}-1\right) \zeta_{\varepsilon}\left(s+\frac{m}{2}-2\right)^{-1} .
\end{gathered}
$$

Here, $\Gamma_{2}(s)$ is the double Gamma function (for definition, we refer to [12] or $\left[\mathbf{7}\right.$, Definition 4.10 , p. 751]), the natural numbers $\nu_{1}, \nu_{2}, \ldots, \nu_{N}$ are the orders of the elliptic fixed points in $X_{K}$ and the integers $\alpha_{l}(m, j)$, $\overline{\alpha_{l}}(m, j) \in\left\{0,1, \ldots, \nu_{j}-1\right\}$ are defined in $(2.1), \zeta_{K}(s)$ is the Dedekind zeta function of $K, \zeta_{\varepsilon}(s):=\left(1-\varepsilon^{-2 s}\right)^{-1}$ and $\varepsilon$ is the fundamental unit of $K$.

Let $m \in 2 \mathbb{N}$. We recall that two Laplacians

(1.5) $\Delta_{0}^{(1)}:=-y_{1}^{2}\left(\frac{\partial^{2}}{\partial x_{1}^{2}}+\frac{\partial^{2}}{\partial y_{1}^{2}}\right), \quad \Delta_{m}^{(2)}:=-y_{2}^{2}\left(\frac{\partial^{2}}{\partial x_{2}^{2}}+\frac{\partial^{2}}{\partial y_{2}^{2}}\right)+i m y_{2} \frac{\partial}{\partial x_{2}}$ are acting on $L_{\mathrm{dis}}^{2}\left(\Gamma_{K} \backslash \mathbb{H}^{2} ;(0, m)\right)$, the space of Hilbert-Maass forms for $\Gamma_{K}$ with weight $(0, m)$. (See Definition 2.6.) We consider a certain subspace of $L_{\text {dis }}^{2}\left(\Gamma_{K} \backslash \mathbb{H}^{2} ;(0, m)\right)$ given by

$$
V_{m}^{(2)}=\left\{f\left(z_{1}, z_{2}\right) \in L_{\mathrm{dis}}^{2}\left(\Gamma_{K} \backslash \mathbb{H}^{2} ;(0, m)\right) \mid \Delta_{m}^{(2)} f=\frac{m}{2}\left(1-\frac{m}{2}\right) f\right\} .
$$

The set of eigenvalues of $\left.\Delta_{0}^{(1)}\right|_{V_{m}^{(2)}}$ are enumerated as

$$
0<\lambda_{0}(m) \leq \lambda_{1}(m) \leq \cdots \leq \lambda_{n}(m) \leq \cdots
$$

Let $s$ be a fixed sufficiently large real number. We consider the spectral zeta function by using these eigenvalues.

$$
\zeta_{m}(w, s)=\sum_{n=0}^{\infty} \frac{1}{\left(\lambda_{n}(m)+s(s-1)\right)^{w}} \quad(\operatorname{Re}(w) \gg 0) .
$$

We can show that $\zeta_{m}(w, s)$ is holomorphic at $w=0$. (See Proposition 4.3.) 
Let us define the regularized determinants of the Laplacian $\left.\Delta_{0}^{(1)}\right|_{V_{m}^{(2)}}$.

Definition 1.5 (Determinants of restrictions of $\left.\Delta_{0}^{(1)}\right)$. Let $m \in 2 \mathbb{N}$. For $s \gg 0$, define

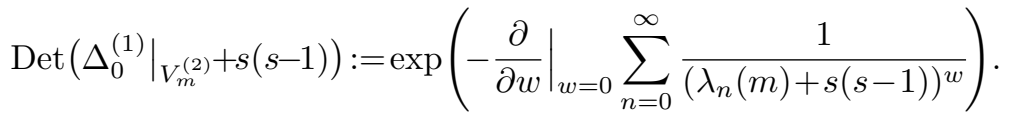

We see later that $\operatorname{Det}\left(\left.\Delta_{0}^{(1)}\right|_{V_{m}^{(2)}}+s(s-1)\right)$ can be extended to an entire function of $s$. (See Corollary 1.7.)

Our main theorem is as follows.

Theorem 1.6 (Main Theorem). Let $\square_{m}:=\left.\Delta_{0}^{(1)}\right|_{V_{m}^{(2)}}$ for $m \in 2 \mathbb{N}$. We have the following determinant expressions of the completed Selberg type zeta functions.

(1) $\widehat{Z}_{2}^{\frac{1}{2}}(s)=e^{\left(s-\frac{1}{2}\right)^{2} \zeta_{K}(-1)+C_{2}} \frac{\operatorname{Det}\left(\square_{2}+s(s-1)\right)}{s(s-1)}$.

(2) $\widehat{Z}_{4}(s)=e^{2\left(s-\frac{1}{2}\right)^{2} \zeta_{K}(-1)+C_{4}} \frac{s(s-1) \operatorname{Det}\left(\square_{4}+s(s-1)\right)}{\operatorname{Det}\left(\square_{2}+s(s-1)\right)}$.

(3) For $m \geq 6, \widehat{Z}_{m}(s)=e^{2\left(s-\frac{1}{2}\right)^{2} \zeta_{K}(-1)+C_{m}} \frac{\operatorname{Det}\left(\square_{m}+s(s-1)\right)}{\operatorname{Det}\left(\square_{m-2}+s(s-1)\right)}$.

Here, the constants $C_{m}$ are given by

$$
\begin{aligned}
C_{2} & =-\frac{1}{2} \log \varepsilon+\sum_{j=1}^{N} \frac{\nu_{j}^{2}-1}{12 \nu_{j}} \log \nu_{j}, \\
C_{m} & =\sum_{j=1}^{N} \frac{\nu_{j}^{2}-1-12 \alpha_{0}(m, j)\left\{\nu_{j}-\alpha_{0}(m, j)\right\}}{6 \nu_{j}} \log \nu_{j} \quad(m \geq 4),
\end{aligned}
$$

the natural numbers $\nu_{1}, \nu_{2}, \ldots, \nu_{N}$ are the orders of the elliptic fixed points in $X_{K}$, and the integers $\alpha_{0}(m, j) \in\left\{0,1, \ldots, \nu_{j}-1\right\}$ are defined in (2.1).

We know the following Weyl's law:

$$
N_{m}^{+}(T):=\#\left\{j \mid \lambda_{j}(m) \leq T\right\} \sim \frac{(m-1)}{2} \zeta_{K}(-1) T \quad(T \rightarrow \infty) .
$$

(See [6, Theorem 6.11].) Therefore, we may say that $Z_{m}(s)(m \geq 4)$ have "more" zeros than poles.

We have several corollaries from Theorem 1.6 by direct calculation. 
Corollary 1.7. Let $\square_{m}=\left.\Delta_{0}^{(1)}\right|_{V_{m}^{(2)}}$ for $m \in 2 \mathbb{N}$. For $m \in 2 \mathbb{N}$, we have

(1) $\operatorname{Det}\left(\square_{2}+s(s-1)\right)=s(s-1) e^{-\left(s-\frac{1}{2}\right)^{2} \zeta_{K}(-1)-C_{2}} \widehat{Z}_{2}^{\frac{1}{2}}(s)$.

(2) $\operatorname{Det}\left(\square_{m}+s(s-1)\right)=e^{-(m-1)\left(s-\frac{1}{2}\right)^{2} \zeta_{K}(-1)-\left(C_{2}+C_{4}+\cdots+C_{m}\right)} \widehat{Z}_{2}^{\frac{1}{2}}(s) \widehat{Z}_{4}(s)$ $\cdots \widehat{Z}_{m}(s)$ for $m \geq 4$.

It follows from the above corollary that $\operatorname{Det}\left(\square_{m}+s(s-1)\right)(m \in 2 \mathbb{N})$ can be extended to entire functions of $s$.

By putting $s=1$ in the above, we have

Corollary 1.8. For $m \in 2 \mathbb{N}$, we have

(1) $\operatorname{Det}\left(\square_{2}\right)=e^{-\frac{1}{4} \zeta_{K}(-1)-C_{2}} \operatorname{Res}_{s=1} \widehat{Z}_{2}^{\frac{1}{2}}(s)$.

(2) $\operatorname{Det}\left(\square_{4}\right)=e^{-\frac{3}{4} \zeta_{K}(-1)-\left(C_{2}+C_{4}\right)} \operatorname{Res}_{s=1} \widehat{Z}_{2}^{\frac{1}{2}}(s) \widehat{Z}_{4}^{\prime}(1)$.

(3) $\operatorname{Det}\left(\square_{m}\right)=e^{-\frac{m-1}{4} \zeta_{K}(-1)-\left(C_{2}+C_{4}+\cdots+C_{m}\right)} \operatorname{Res}_{s=1} \widehat{Z}_{2}^{\frac{1}{2}}(s) \widehat{Z}_{4}^{\prime}(1) \widehat{Z}_{6}(1)$ ... $\widehat{Z}_{m}(1)$ for $m \geq 6$.

Here, $\square_{m}=\left.\Delta_{0}^{(1)}\right|_{V_{m}^{(2)}}$ for $m \in 2 \mathbb{N}$.

Finally, we have a few comments on related works. Let $Z_{Y}(s)$ be the Selberg zeta function for a modular curve $Y$. As kindly pointed out by the referee, the special value $Z_{Y}^{\prime}(1)$ is evaluated by an arithmetic Riemann-Roch formula in the realm of Arakelov geometry in $[\mathbf{3}, \mathbf{4}]$. Therefore, we might imagine that our results on regularized determinants and special Selberg type zeta values should play the role of the missing holomorphic analytic torsion of sheaves of higher weight Hilbert modular forms, in a conjectural arithmetic Riemann-Roch formula à la GilletSoulé.

For this reason, to work out the case for congruence subgroups of $\Gamma_{K}$ of a quadratic field $K$ with arbitrary class number would be interesting and make the range of application wider. We hope to treat this problem in a future paper since our results depend on "explicit Selberg trace formulas" for $\Gamma_{K}$ with class number one in $[6]$.

\section{Preliminaries}

We fix the notation for the Hilbert modular group of a real quadratic field in this section. We also recall the definition of Hilbert-Maass forms for the Hilbert modular group and review "Differences of the Selberg trace formula", introduced in [6], which play a crucial role in this article.

2.1. Hilbert modular group of a real quadratic field. Let $K / \mathbb{Q}$ be a real quadratic field with class number one and $\mathcal{O}_{K}$ be the ring of integers of $K$. Put $D$ be the discriminant of $K$ and $\varepsilon>1$ be the fundamental 
unit of $K$. We denote the generator of $\operatorname{Gal}(K / \mathbb{Q})$ by $\sigma$ and put $a^{\prime}:=\sigma(a)$ for $a \in K$. We also put $\gamma^{\prime}=\left(\begin{array}{ll}a^{\prime} & b^{\prime} \\ c^{\prime} & d^{\prime}\end{array}\right)$ for $\gamma=\left(\begin{array}{ll}a & b \\ c & d\end{array}\right) \in \operatorname{PSL}\left(2, \mathcal{O}_{K}\right)$.

Let $G$ be $\operatorname{PSL}(2, \mathbb{R})^{2}=(\operatorname{SL}(2, \mathbb{R}) /\{ \pm I\})^{2}$ and $\mathbb{H}^{2}$ be the direct product of two copies of the upper half plane $\mathbb{H}:=\{z \in \mathbb{C} \mid \operatorname{im}(z)>0\}$. The group $G$ acts on $\mathbb{H}^{2}$ by

$$
g . z=\left(g_{1}, g_{2}\right) \cdot\left(z_{1}, z_{2}\right)=\left(\frac{a_{1} z_{1}+b_{1}}{c_{1} z_{1}+d_{1}}, \frac{a_{2} z_{2}+b_{2}}{c_{2} z_{2}+d_{2}}\right) \in \mathbb{H}^{2}
$$

for $g=\left(g_{1}, g_{2}\right)=\left(\left(\begin{array}{ll}a_{1} & b_{1} \\ c_{1} & d_{1}\end{array}\right),\left(\begin{array}{ll}a_{2} & b_{2} \\ c_{2} & d_{2}\end{array}\right)\right)$ and $z=\left(z_{1}, z_{2}\right) \in \mathbb{H}^{2}$.

A discrete subgroup $\Gamma \subset G$ is called irreducible if it is not commensurable with any direct product $\Gamma_{1} \times \Gamma_{2}$ of two discrete subgroups of $\operatorname{PSL}(2, \mathbb{R})$. We have classification of the elements of irreducible $\Gamma$.

Proposition 2.1 (Classification of the elements). Let $\Gamma$ be an irreducible discrete subgroup of $G$. Then any element of $\Gamma$ is one of the following:

(1) $\gamma=(I, I)$ is the identity.

(2) $\gamma=\left(\gamma_{1}, \gamma_{2}\right)$ is hyperbolic $\Leftrightarrow\left|\operatorname{tr}\left(\gamma_{1}\right)\right|>2$ and $\left|\operatorname{tr}\left(\gamma_{2}\right)\right|>2$.

(3) $\gamma=\left(\gamma_{1}, \gamma_{2}\right)$ is elliptic $\Leftrightarrow\left|\operatorname{tr}\left(\gamma_{1}\right)\right|<2$ and $\left|\operatorname{tr}\left(\gamma_{2}\right)\right|<2$.

(4) $\gamma=\left(\gamma_{1}, \gamma_{2}\right)$ is hyperbolic-elliptic $\Leftrightarrow\left|\operatorname{tr}\left(\gamma_{1}\right)\right|>2$ and $\left|\operatorname{tr}\left(\gamma_{2}\right)\right|<2$.

(5) $\gamma=\left(\gamma_{1}, \gamma_{2}\right)$ is elliptic-hyperbolic $\Leftrightarrow\left|\operatorname{tr}\left(\gamma_{1}\right)\right|<2$ and $\left|\operatorname{tr}\left(\gamma_{2}\right)\right|>2$.

(6) $\gamma=\left(\gamma_{1}, \gamma_{2}\right)$ is parabolic $\Leftrightarrow\left|\operatorname{tr}\left(\gamma_{1}\right)\right|=\left|\operatorname{tr}\left(\gamma_{2}\right)\right|=2$.

Note that there are no other types in $\Gamma$ (parabolic-elliptic, etc.).

Let us consider the Hilbert modular group of the real quadratic field $K$ with class number one,

$$
\Gamma_{K}:=\left\{\left(\gamma, \gamma^{\prime}\right)=\left(\left(\begin{array}{ll}
a & b \\
c & d
\end{array}\right),\left(\begin{array}{ll}
a^{\prime} & b^{\prime} \\
c^{\prime} & d^{\prime}
\end{array}\right)\right) \mid\left(\begin{array}{ll}
a & b \\
c & d
\end{array}\right) \in \operatorname{PSL}\left(2, \mathcal{O}_{K}\right)\right\} .
$$

It is known that $\Gamma_{K}$ is an irreducible discrete subgroup of $G=$ $\operatorname{PSL}(2, \mathbb{R})^{2}$ with the only one cusp $\infty:=(\infty, \infty)$, i.e. $\Gamma_{K}$-inequivalent parabolic fixed point. $X_{K}=\Gamma_{K} \backslash \mathbb{H}^{2}$ is called the Hilbert modular surface.

We have a lemma about the Euler characteristic of the Hilbert modular surface $X_{K}$.

Lemma 2.2. Let $E\left(X_{K}\right)$ be the Euler characteristic of the Hilbert modular surface $X_{K}=\Gamma_{K} \backslash \mathbb{H}^{2}$. Then we have $E\left(X_{K}\right) \in 2 \mathbb{N}$.

Proof: By noting the formula $E\left(X_{K}\right)=2 \zeta_{K}(-1)+\sum_{j=1}^{N} \frac{\nu_{j}-1}{\nu_{j}}$ (see (2), (4) in [9, pp. 46-47]), $E\left(X_{K}\right)$ is a positive integer. Let $Y_{K}$ and $Y_{K}^{-}$be the non-singular algebraic surfaces resolved singularities, in the canonical 
minimal way, of compactifications of $\Gamma_{K} \backslash \mathbb{H}^{2}$ and $\Gamma_{K} \backslash\left(\mathbb{H} \times \mathbb{H}^{-}\right)$respectively. Here $\mathbb{H}^{-}$is the lower half plane. Let $\chi\left(Y_{K}\right)$ and $\chi\left(Y_{K}^{-}\right)$be the arithmetic genera of $Y_{K}$ and $Y_{K}^{-}$respectively. By formulas (12) and (14) in $[\mathbf{9}$, p. 48], we have

$$
E\left(X_{K}\right)=2\left(\chi\left(Y_{K}\right)+\chi\left(Y_{K}^{-}\right)\right) .
$$

We complete the proof.

We fix the notation for elliptic conjugacy classes in $\Gamma_{K}$. Let $R_{1}, R_{2}, \ldots$, $R_{N}$ be a complete system of representatives of the $\Gamma_{K}$-conjugacy classes of primitive elliptic elements of $\Gamma_{K} . \nu_{1}, \nu_{2}, \ldots, \nu_{N}\left(\nu_{j} \in \mathbb{N}, \nu_{j} \geq 2\right)$ denote the orders of $R_{1}, R_{2}, \ldots, R_{N}$. We may assume that $R_{j}$ is conjugate in $\operatorname{PSL}(2, \mathbb{R})^{2}$ to

$$
R_{j} \sim\left(\left(\begin{array}{cc}
\cos \frac{\pi}{\nu_{j}} & -\sin \frac{\pi}{\nu_{j}} \\
\sin \frac{\pi}{\nu_{j}} & \cos \frac{\pi}{\nu_{j}}
\end{array}\right),\left(\begin{array}{cc}
\cos \frac{t_{j} \pi}{\nu_{j}} & -\sin \frac{t_{j} \pi}{\nu_{j}} \\
\sin \frac{t_{j} \pi}{\nu_{j}} & \cos \frac{t_{j} \pi}{\nu_{j}}
\end{array}\right)\right), \quad\left(t_{j}, \nu_{j}\right)=1 .
$$

For an even natural number $m \geq 2$ and $l \in\left\{0,1, \ldots, \nu_{j}-1\right\}$, we define $\alpha_{l}(m, j), \overline{\alpha_{l}}(m, j) \in\left\{0,1, \ldots, \nu_{j}-1\right\}$ by

$$
\begin{aligned}
& l+\frac{t_{j}(m-2)}{2} \equiv \alpha_{l}(m, j) \quad\left(\bmod \nu_{j}\right), \\
& l-\frac{t_{j}(m-2)}{2} \equiv \overline{\alpha_{l}}(m, j) \quad\left(\bmod \nu_{j}\right) .
\end{aligned}
$$

We divide hyperbolic conjugacy classes of $\Gamma_{K}$ into two subclasses according to their types.

Definition 2.3 (Types of hyperbolic elements). For a hyperbolic element $\gamma$, we define that:

(1) $\gamma$ is type 1 hyperbolic $\Leftrightarrow$ whose all fixed points are not fixed by parabolic elements.

(2) $\gamma$ is type 2 hyperbolic $\Leftrightarrow$ not type 1 hyperbolic.

We denote by $\Gamma_{\mathrm{H} 1}, \Gamma_{\mathrm{E}}, \Gamma_{\mathrm{HE}}, \Gamma_{\mathrm{EH}}$, and $\Gamma_{\mathrm{H} 2}$, type 1 hyperbolic $\Gamma_{K}$-conjugacy classes, elliptic $\Gamma_{K}$-conjugacy classes, hyperbolic-elliptic $\Gamma_{K}$-conjugacy classes, elliptic-hyperbolic $\Gamma_{K}$-conjugacy classes and type 2 hyperbolic $\Gamma_{K}$-conjugacy classes of $\Gamma_{K}$ respectively.

2.2. The space of Hilbert-Maass forms. Fix the weight $\left(m_{1}, m_{2}\right) \in$ $(2 \mathbb{Z})^{2}$. Set the automorphic factor $j_{\gamma}\left(z_{j}\right)=\frac{c z_{j}+d}{\left|c z_{j}+d\right|}$ for $\gamma \in \operatorname{PSL}(2, \mathbb{R})$ $(j=1,2)$.

Let $\Delta_{m_{j}}^{(j)}:=-y_{j}^{2}\left(\frac{\partial^{2}}{\partial x_{j}^{2}}+\frac{\partial^{2}}{\partial y_{j}^{2}}\right)+i m_{j} y_{j} \frac{\partial}{\partial x_{j}}(j=1,2)$ be the Laplacians of weight $m_{j}$ for the variable $z_{j}$. 
Let us define the $L^{2}$-space of automorphic forms of weight $\left(m_{1}, m_{2}\right)$ with respect to the Hilbert modular group $\Gamma_{K}$.

Definition 2.4 ( $L^{2}$-space of automorphic forms of weight $\left.\left(m_{1}, m_{2}\right)\right)$. $L^{2}\left(\Gamma_{K} \backslash \mathbb{H}^{2} ;\left(m_{1}, m_{2}\right)\right)$ is defined to be the set of functions $f: \mathbb{H}^{2} \rightarrow \mathbb{C}$, of class $C^{\infty}$ satisfying:

(i) $f\left(\left(\gamma, \gamma^{\prime}\right)\left(z_{1}, z_{2}\right)\right)=j_{\gamma}\left(z_{1}\right)^{m_{1}} j_{\gamma^{\prime}}\left(z_{2}\right)^{m_{2}} f\left(z_{1}, z_{2}\right), \forall\left(\gamma, \gamma^{\prime}\right) \in \Gamma_{K}$;

(ii) $\exists\left(\lambda^{(1)}, \lambda^{(2)}\right) \in \mathbb{R}^{2}$ such that

$$
\Delta_{m_{1}}^{(1)} f\left(z_{1}, z_{2}\right)=\lambda^{(1)} f\left(z_{1}, z_{2}\right), \quad \Delta_{m_{2}}^{(2)} f\left(z_{1}, z_{2}\right)=\lambda^{(2)} f\left(z_{1}, z_{2}\right) ;
$$

(iii) $\|f\|^{2}=\int_{\Gamma_{K} \backslash \mathbb{H}^{2}} f(z) \overline{f(z)} d \mu(z)<\infty$.

Here, $d \mu(z)=\frac{d x_{1} d y_{1}}{y_{1}^{2}} \frac{d x_{2} d y_{2}}{y_{2}^{2}}$ for $z=\left(z_{1}, z_{2}\right) \in \mathbb{H}^{2}$.

Then, it is known that:

Proposition 2.5. Let $L_{\mathrm{dis}}^{2}\left(\Gamma_{K} \backslash \mathbb{H}^{2} ;\left(m_{1}, m_{2}\right)\right)$ be the subspace of the discrete spectrum of the Laplacians and $L_{\text {con }}^{2}\left(\Gamma_{K} \backslash \mathbb{H}^{2} ;\left(m_{1}, m_{2}\right)\right)$ be the subspace of the continuous spectrum. Then, we have a direct sum decomposition:

$L^{2}\left(\Gamma_{K} \backslash \mathbb{H}^{2} ;\left(m_{1}, m_{2}\right)\right)=L_{\text {dis }}^{2}\left(\Gamma_{K} \backslash \mathbb{H}^{2} ;\left(m_{1}, m_{2}\right)\right) \oplus L_{\text {con }}^{2}\left(\Gamma_{K} \backslash \mathbb{H}^{2} ;\left(m_{1}, m_{2}\right)\right)$

and there is an orthonormal basis $\left\{\phi_{j}\right\}_{j=0}^{\infty}$ of $L_{\mathrm{dis}}^{2}\left(\Gamma_{K} \backslash \mathbb{H}^{2} ;\left(m_{1}, m_{2}\right)\right)$.

Definition 2.6 (Hilbert-Maass forms of weight $\left.\left(m_{1}, m_{2}\right)\right)$. Let $\left(m_{1}, m_{2}\right) \in$ $(2 \mathbb{Z})^{2}$. We call

$$
L_{\text {dis }}^{2}\left(\Gamma_{K} \backslash \mathbb{H}^{2} ;\left(m_{1}, m_{2}\right)\right)
$$

the space of Hilbert-Maass forms for $\Gamma_{K}$ of weight $\left(m_{1}, m_{2}\right)$.

Let $\left\{\phi_{j}\right\}_{j=0}^{\infty}$ be an orthonormal basis of $L_{\text {dis }}^{2}\left(\Gamma_{K} \backslash \mathbb{H}^{2} ;\left(m_{1}, m_{2}\right)\right)$ and $\left(\lambda_{j}^{(1)}, \lambda_{j}^{(2)}\right) \in \mathbb{R}^{2}$ such that

$$
\Delta_{m_{1}}^{(1)} \phi_{j}=\lambda_{j}^{(1)} \phi_{j} \quad \text { and } \quad \Delta_{m_{2}}^{(2)} \phi_{j}=\lambda_{j}^{(2)} \phi_{j} .
$$

We write $\lambda_{j}^{(l)}=\frac{1}{4}+\left(r_{j}^{(l)}\right)^{2}$ and $r_{j}^{(i)}$ are defined by

$$
r_{j}^{(l)}:= \begin{cases}\sqrt{\lambda_{j}^{(l)}-\frac{1}{4}} & \text { if } \lambda_{j}^{(l)} \geq \frac{1}{4}, \\ i \sqrt{\frac{1}{4}-\lambda_{j}^{(l)}} & \text { if } \lambda_{j}^{(l)}<\frac{1}{4},\end{cases}
$$

for $l=1,2$. 
2.3. Double differences of the Selberg trace formula. Let $m$ be an even integer. We studied and derived the full Selberg trace formula for $L^{2}\left(\Gamma_{K} \backslash \mathbb{H}^{2} ;(0, m)\right)$ in $[6]$. Let $h\left(r_{1}, r_{2}\right)$ be an even "test function" which satisfy certain analytic conditions. Roughly speaking, [6, Theorem 2.22] is as follows:

$$
\sum_{j=0}^{\infty} h\left(r_{j}^{(1)}, r_{j}^{(2)}\right)=\mathbf{I}(h)+\mathbf{I I}_{\mathbf{a}}(h)+\mathbf{I I}_{\mathbf{b}}(h)+\mathbf{I I I}(h) .
$$

Here, the right hand side is a sum of distributions of $h$ contributed from several conjugacy classes of $\Gamma_{K}$ and Eisenstein series for $\Gamma_{K}$. Assuming that the test function $h\left(r_{1}, r_{2}\right)$ is a product of $h_{1}\left(r_{1}\right)$ and $h_{2}\left(r_{2}\right)$, we derived "differences of STF" [6, Theorem 4.1] and "double differences of STF" [6, Theorem 4.4]. We explain for this.

Let us consider the subspace of $L_{\text {dis }}^{2}\left(\Gamma_{K} \backslash \mathbb{H}^{2} ;(0, m)\right)$ given by

$$
V_{m}^{(2)}=\left\{f \in L_{\mathrm{dis}}^{2}\left(\Gamma_{K} \backslash \mathbb{H}^{2} ;(0, m)\right) \mid \Delta_{m}^{(2)} f=\frac{m}{2}\left(1-\frac{m}{2}\right) f\right\} .
$$

Let $h_{1}(r)$ be an even function, analytic in $\operatorname{im}(r)<\delta$ for some $\delta>0$,

$$
h_{1}(r)=O\left(\left(1+|r|^{2}\right)^{-2-\delta}\right)
$$

for some $\delta>0$ in this domain. Let $g_{1}(u):=\frac{1}{2 \pi} \int_{-\infty}^{\infty} h_{1}(r) e^{-i r u} d r$. Then we have

Proposition 2.7 (Double differences of STF for $\left.L^{2}\left(\Gamma_{K} \backslash \mathbb{H}^{2} ;(0,2)\right)\right)$. Let $m=2$. We have

$$
\begin{aligned}
\sum_{j=0}^{\infty} h_{1}\left(\rho_{j}(2)\right)-h_{1}\left(\frac{i}{2}\right) & \operatorname{vol}\left(\Gamma_{K} \backslash \mathbb{H}^{2}\right) \\
= & \int_{-\infty}^{\infty} r h_{1}(r) \tanh (\pi r) d r \\
& -\sum_{R\left(\theta_{1}, \theta_{2}\right) \in \Gamma_{\mathrm{E}}} \frac{i e^{-i \theta_{1}}}{8 \nu_{R} \sin \theta_{1}} \int_{-\infty}^{\infty} g_{1}(u) e^{-u / 2}\left[\frac{e^{u}-e^{2 i \theta_{1}}}{\cosh u-\cos 2 \theta_{1}}\right] d u \\
& -\frac{1}{2} \sum_{(\gamma, \omega) \in \Gamma_{\mathrm{HE}}} \frac{\log N\left(\gamma_{0}\right) g_{1}(\log N(\gamma))}{N(\gamma)^{1 / 2}-N(\gamma)^{-1 / 2}}-\log \varepsilon g_{1}(0) \\
& -2 \log \varepsilon \sum_{k=1}^{\infty} g_{1}(2 k \log \varepsilon) \varepsilon^{-k} .
\end{aligned}
$$

Here, $\left\{\lambda_{j}(2)=1 / 4+\rho_{j}(2)^{2}\right\}_{j=0}^{\infty}$ is the set of eigenvalues of the Laplacian $\Delta_{0}^{(1)}$ acting on $V_{2}^{(2)}$. 
Proof: See [6, Corollary 6.3].

Proposition 2.8 (Double differences of STF for $L^{2}\left(\Gamma_{K} \backslash \mathbb{H}^{2} ;(0, m)\right)$ ). Let $m \in 2 \mathbb{N}$ and $m \geq 4$. We have

$$
\begin{aligned}
\sum_{j=0}^{\infty} h_{1}\left(\rho_{j}(m)\right)-\sum_{j=0}^{\infty} h_{1}\left(\rho_{j}(m-2)\right)+\delta_{m, 4} h_{1}\left(\frac{i}{2}\right) \\
=\frac{\operatorname{vol}\left(\Gamma_{K} \backslash \mathbb{H}^{2}\right)}{8 \pi^{2}} \int_{-\infty}^{\infty} r h_{1}(r) \tanh (\pi r) d r \\
\quad-\sum_{R\left(\theta_{1}, \theta_{2}\right) \in \Gamma_{\mathrm{E}}} \frac{i e^{-i \theta_{1}} e^{i(m-2) \theta_{2}}}{4 \nu_{R} \sin \theta_{1}} \int_{-\infty}^{\infty} g_{1}(u) e^{-u / 2}\left[\frac{e^{u}-e^{2 i \theta_{1}}}{\cosh u-\cos 2 \theta_{1}}\right] d u \\
-\sum_{(\gamma, \omega) \in \Gamma_{\mathrm{HE}}} \frac{\log N(\gamma(\gamma)}{N(\gamma)^{1 / 2}-N(\gamma)^{-1 / 2}} g_{1}(\log N(\gamma)) e^{i(m-2) \omega} \\
-2 \log \varepsilon \sum_{k=1}^{\infty} g_{1}(2 k \log \varepsilon)\left(\varepsilon^{-k(m-1)}-\varepsilon^{-k(m-3)}\right) .
\end{aligned}
$$

Here, $\left\{\lambda_{j}(q)=1 / 4+\rho_{j}(q)^{2}\right\}_{j=0}^{\infty}$ is the set of eigenvalues of the Laplacian $\Delta_{0}^{(1)}$ acting on $V_{q}^{(2)}(q=m, m-2)$.

Proof: See [6, Theorem 4.4] and [6, (5.3)].

\section{Asymptotic behavior of the completed Selberg zeta functions}

We have to know the asymptotic behavior of the completed Selberg zeta functions $\widehat{Z}_{2}^{\frac{1}{2}}(s)$ and $\widehat{Z}_{m}(s)(m \geq 4)$ when $s \rightarrow \infty$, to prove Main Theorem (Theorem 1.6). We calculate their asymptotic behavior in this section.

Lemma 3.1 (Stirling's formula for $\left.\Gamma_{2}(z)\right)$. We have

$$
\log \Gamma_{2}(z+1)=\frac{3}{4} z^{2}-\left(\frac{z^{2}}{2}-\frac{1}{12}\right) \log z+o(1) \quad(z \rightarrow \infty),
$$

where $\Gamma_{2}(z):=\exp \left(\left.\frac{\partial}{\partial s}\right|_{s=0} \sum_{m, n=0}^{\infty}(m+n+z)^{-s}\right)$ denotes the double Gamma function.

Proof: Let $G(z)$ be the Barnes $G$-function defined by

$$
G(z+1)=(2 \pi)^{\frac{z}{2}} e^{-\frac{z+z^{2}(1+\gamma)}{2}} \prod_{k=1}^{\infty}\left\{\left(1+\frac{z}{k}\right)^{k} e^{-z+\frac{z^{2}}{k^{2}}}\right\} .
$$


(See $\left[\mathbf{1}\right.$, p. 268].) Here, $\gamma=-\Gamma^{\prime}(1)$ is the Euler constant. By using the relation

$$
\Gamma_{2}(z)=e^{\zeta^{\prime}(-1)}(2 \pi)^{\frac{z-1}{2}} G(z)^{-1}
$$

(see [14, Proposition 4.1]) and the asymptotic formula $\log G(z+1)=\frac{z}{2} \log (2 \pi)+\zeta^{\prime}(-1)-\frac{3}{4} z^{2}+\left(\frac{z^{2}}{2}-\frac{1}{12}\right) \log z+o(1) \quad(z \rightarrow \infty)$, (see [1, p. 269]) we have the desired formula.

Lemma 3.2 (Asymptotics of the identity factors). We have

(3.2) $\log Z_{\mathrm{id}}^{\frac{1}{2}}(s)=\zeta_{K}(-1)\left\{\frac{3}{2} s^{2}-s-\left(s^{2}-s+\frac{1}{3}\right) \log s\right\}+o(1) \quad(s \rightarrow \infty)$,

(3.3) $\log Z_{\mathrm{id}}(s)=2 \zeta_{K}(-1)\left\{\frac{3}{2} s^{2}-s-\left(s^{2}-s+\frac{1}{3}\right) \log s\right\}+o(1)(s \rightarrow \infty)$.

Proof: By Definition 1.4,

$$
\log Z_{\mathrm{id}}^{\frac{1}{2}}(s)=\zeta_{K}(-1)\left(\log \Gamma_{2}(s)+\log \Gamma_{2}(s+1)\right)
$$

and Lemma 3.1, we have the desired (3.2). We see that the relation $\log Z_{\text {id }}(s)=2 \log Z_{\text {id }}^{\frac{1}{2}}(s)$ implies (3.3). It completes the proof.

Lemma 3.3 (Asymptotics of the elliptic factors). We have

(3.4) $\log Z_{\mathrm{ell}}^{\frac{1}{2}}(s ; 2)=-\sum_{j=1}^{N} \frac{\nu_{j}^{2}-1}{12 \nu_{j}} \log \frac{s}{\nu_{j}}+o(1) \quad(s \rightarrow \infty)$,

(3.5) $\log Z_{\mathrm{ell}}(s ; m)$

$$
=-\sum_{j=1}^{N} \frac{\nu_{j}^{2}-1-12 \alpha_{0}(m, j)\left\{\nu_{j}-\alpha_{0}(m, j)\right\}}{6 \nu_{j}} \log \frac{s}{\nu_{j}}+o(1)(s \rightarrow \infty)
$$

for $m \in 2 \mathbb{N}$ and $m \geq 4$. Here $\alpha_{0}(m, j) \in\left\{0,1, \ldots, \nu_{j}-1\right\}$ are defined in (2.1).

Proof: We use Stirling's formula of $\Gamma(z)$ (see [13, p. 12]):

$$
\log \Gamma(z)=\left(z-\frac{1}{2}\right) \log z-z+\frac{1}{2} \log (2 \pi)+o(1) \quad(z \rightarrow \infty) .
$$


By Definition 1.4,

$$
\log Z_{\mathrm{ell}}(s ; m)=\sum_{j=1}^{N} \sum_{l=0}^{\nu_{j}-1} \frac{\nu_{j}-1-\alpha_{l}(m, j)-\overline{\alpha_{l}}(m, j)}{\nu_{j}} \log \Gamma\left(\frac{s+l}{\nu_{j}}\right) .
$$

We see that $\left\{\alpha_{l}(m, j) \mid 0 \leq l \leq \nu_{j}-1\right\}=\left\{\bar{\alpha}_{l}(m, j) \mid 0 \leq l \leq \nu_{j}-1\right\}=$ $\left\{0,1,2, \ldots, \nu_{j}-1\right\}$ for each $j$. Thus we have $\sum_{l=0}^{\nu_{j}-1}\left(\nu_{j}-1-\alpha_{l}(m, j)-\right.$ $\left.\overline{\alpha_{l}}(m, j)\right)=0$, and find that

$$
\begin{aligned}
& \sum_{l=0}^{\nu_{j}-1} \frac{\nu_{j}-1-\alpha_{l}(m, j)-\overline{\alpha_{l}}(m, j)}{\nu_{j}} \log \Gamma\left(\frac{s+l}{\nu_{j}}\right) \\
& =\sum_{l=0}^{\nu_{j}-1} \frac{\nu_{j}-1-\alpha_{l}(m, j)-\overline{\alpha_{l}}(m, j)}{\nu_{j}} \\
& \times\left\{\left(\frac{s+l}{\nu_{j}}-\frac{1}{2}\right) \log \left(\frac{s+l}{\nu_{j}}\right)-\frac{s+l}{\nu_{j}}+\frac{1}{2} \log (2 \pi)\right\}+o(1) \\
& =\sum_{l=0}^{\nu_{j}-1} \frac{\nu-1-\alpha_{l}(m, j)-\overline{\alpha_{l}}(m, j)}{\nu_{j}} \\
& \times\left\{\left(\frac{s+l}{\nu_{j}}-\frac{1}{2}\right) \log (s+l)-\frac{l}{\nu} \log \nu_{j}-\frac{l}{\nu_{j}}\right\}+o(1) \\
& =\sum_{l=0}^{\nu_{j}-1} \frac{\nu_{j}-1-\alpha_{l}(m, j)-\overline{\alpha_{l}}(m, j)}{\nu_{j}}\left\{\left(\frac{s}{\nu_{j}}-\frac{1}{2}\right) \log s+\frac{l}{\nu_{j}} \log \frac{s}{\nu_{j}}\right\}+o(1) \\
& =\sum_{l=0}^{\nu_{j}-1} \frac{\nu_{j}-1-\alpha_{l}(m, j)-\overline{\alpha_{l}}(m, j)}{\nu_{j}} \frac{l}{\nu_{j}} \log \frac{s}{\nu_{j}}+o(1) \\
& =\frac{\left(\nu_{j}-1\right)^{2}}{2 \nu_{j}} \log \frac{s}{\nu_{j}}-\sum_{l=0}^{\nu_{j}-1} \frac{\alpha_{l}(m, j)+\overline{\alpha_{l}}(m, j)}{\nu_{j}} \frac{l}{\nu_{j}} \log \frac{s}{\nu_{j}}+o(1) \\
& (s \rightarrow \infty) \text {. }
\end{aligned}
$$

By (2.1), we can check that

$$
\alpha_{l}(m, j)= \begin{cases}\alpha_{0}(m, j)+l & \left(0 \leq l \leq \nu_{j}-\alpha_{0}(m, j)-1\right), \\ \alpha_{0}(m, j)-\nu_{j}+l & \left(\nu_{j}-\alpha_{0}(m, j) \leq l \leq \nu_{j}-1\right),\end{cases}
$$


hence we calculate further,

$$
\begin{aligned}
\sum_{l=0}^{\nu_{j}-1} \frac{l \alpha_{l}(m, j)}{\nu_{j}^{2}}= & \sum_{l=0}^{\nu_{j}-\alpha_{0}(m, j)-1} \frac{l\left(\alpha_{0}(m, j)+l\right)}{\nu_{j}^{2}} \\
& +\sum_{l=\nu_{j}-\alpha_{0}(m, j)}^{\nu_{j}-1} \frac{l\left(\alpha_{0}(m, j)-\nu_{j}+l\right)}{\nu_{j}^{2}} \\
= & \frac{\left(\nu_{j}-1\right)\left(2 \nu_{j}-1\right)}{6 \nu_{j}}+\frac{\alpha_{0}(m, j)\left(\alpha_{0}(m, j)-\nu_{j}\right)}{\nu_{j}} .
\end{aligned}
$$

By noting $\alpha_{0}(m, j)\left(\alpha_{0}(m, j)-\nu_{j}\right)=\overline{\alpha_{0}}(m, j)\left(\overline{\alpha_{0}}(m, j)-\nu_{j}\right)$, we have

$$
\begin{aligned}
\sum_{l=0}^{\nu_{j}-1} & \frac{\nu_{j}-1-\alpha_{l}(m, j)-\overline{\alpha_{l}}(m, j)}{\nu_{j}} \log \Gamma\left(\frac{s+l}{\nu_{j}}\right) \\
= & \frac{\left(\nu_{j}-1\right)^{2}}{2 \nu_{j}} \log \frac{s}{\nu_{j}}-\sum_{l=0}^{\nu_{j}-1} \frac{\alpha_{l}(m, j)+\overline{\alpha_{l}}(m, j)}{\nu_{j}} \frac{l}{\nu_{j}} \log \frac{s}{\nu_{j}}+o(1) \\
= & \frac{\left(\nu_{j}-1\right)^{2}}{2 \nu_{j}} \log \frac{s}{\nu_{j}} \\
& -2\left\{\frac{\left(\nu_{j}-1\right)\left(2 \nu_{j}-1\right)}{6 \nu_{j}}+\frac{\alpha_{0}(m, j)\left(\alpha_{0}(m, j)-\nu_{j}\right)}{\nu_{j}}\right\} \log \frac{s}{\nu_{j}}+o(1) \\
= & -\frac{\nu_{j}^{2}-1-12 \alpha_{0}(m, j)\left\{\nu_{j}-\alpha_{0}(m, j)\right\}}{6 \nu_{j}} \log \frac{s}{\nu_{j}}+o(1) \quad(s \rightarrow \infty) .
\end{aligned}
$$

Thus we have (3.5). In addition, we note that

$$
\log Z_{\mathrm{ell}}^{\frac{1}{2}}(s ; 2)=\left.\frac{1}{2} \log Z_{\mathrm{ell}}(s ; m)\right|_{m=2} .
$$

Since $\alpha_{l}(2, j)=l$, we see that $\alpha_{0}(2, j)=0$ for any $j$. Therefore we have (3.4). It completes the proof. 
Proposition 3.4 (Asymptotics of the completed Selberg zeta functions). We have

$$
\begin{aligned}
& \log \widehat{Z}_{2}^{\frac{1}{2}}(s)= \zeta_{K}(-1)\left\{\frac{3}{2} s^{2}-s-\left(s^{2}-s+\frac{1}{3}\right) \log s\right\} \\
&-\sum_{j=1}^{N} \frac{\nu_{j}^{2}-1}{12 \nu_{j}} \log \frac{s}{\nu_{j}}-s \log \varepsilon+o(1) \quad(s \rightarrow \infty) \\
& \log \widehat{Z}_{m}(s)= 2 \zeta_{K}(-1)\left\{\frac{3}{2} s^{2}-s-\left(s^{2}-s+\frac{1}{3}\right) \log s\right\} \\
&-\sum_{j=1}^{N} \frac{\nu_{j}^{2}-1-12 \alpha_{0}(m, j)\left\{\nu_{j}-\alpha_{0}(m, j)\right\}}{6 \nu_{j}} \log \frac{s}{\nu_{j}}+o(1) \\
& \quad(s \rightarrow \infty)
\end{aligned}
$$

for $m \in 2 \mathbb{N}$ and $m \geq 4$. Here $\alpha_{0}(m, j) \in\left\{0,1, \ldots, \nu_{j}-1\right\}$ are defined in (2.1).

Proof: We note that $\log \sqrt{Z_{2}(s)}, \log Z_{m}(s)=o(1)(s \rightarrow \infty)$. By Definition 1.4 and Lemmas 3.2 and 3.3, we complete the proof.

\section{Asymptotic behavior of the regularized determinants}

To investigate the analytic nature of the spectral zeta function $\zeta_{m}(w, s)$ at $w=0$, we introduce the theta function $\theta_{m}(t)$ in this section. Since the regularized determinants of the Laplacians $\operatorname{Det}\left(\square_{m}+s(s-1)\right)$ are defined by the derivative of $-\zeta_{m}(w, s)$ at $w=0$, we need to know the asymptotics of $-\left.\frac{\partial}{\partial w} \zeta_{m}(w, s)\right|_{w=0}$ when $s \rightarrow \infty$. We calculate their asymptotics in this section.

Definition 4.1. For $m \in 2 \mathbb{N}$ and $t>0$, define

$$
\theta_{m}(t):=\sum_{j=0}^{\infty} e^{-t \lambda_{j}(m)}
$$

We investigate the asymptotic behavior of $\theta_{m}(t)$ as $t \rightarrow+0$ by using Propositions 2.7 and 2.8, which are called "Double differences of the Selberg trace formula for Hilbert modular surfaces" introduced and proved in [6]. 
Proposition 4.2. We have the following asymptotic formulas.

$$
\begin{aligned}
\theta_{2}(t)= & \frac{1}{2} \zeta_{K}(-1) \frac{1}{t}-\frac{\log \varepsilon}{2 \sqrt{\pi}} \frac{1}{\sqrt{t}} \\
& +\left(-\frac{1}{6} \zeta_{K}(-1)+b_{0}(2)+1\right)+o(1) \quad(t \rightarrow+0), \\
\theta_{m}(t)= & \frac{m-1}{2} \zeta_{K}(-1) \frac{1}{t}-\frac{\log \varepsilon}{2 \sqrt{\pi}} \frac{1}{\sqrt{t}} \\
+ & \left(-\frac{m-1}{6} \zeta_{K}(-1)+b_{0}(2)+b_{0}(4)+\cdots+b_{0}(m)\right)+o(1) \\
& (t \rightarrow+0), \quad(m \in 2 \mathbb{N}, m \geq 4) .
\end{aligned}
$$

Here,

$$
\begin{aligned}
b_{0}(2) & =-\sum_{j=1}^{N} \frac{\nu_{j}^{2}-1}{24 \nu_{j}}, \\
b_{0}(m) & =-\sum_{j=1}^{N} \frac{\nu_{j}^{2}-1-12 \alpha_{0}(m, j)\left\{\nu_{j}-\alpha_{0}(m, j)\right\}}{12 \nu_{j}} \quad(m \geq 4) .
\end{aligned}
$$

Proof: For $t>0$, let us take the pair of test functions $h_{1}(r)=e^{-t\left(r^{2}+1 / 4\right)}$ and $g_{1}(u)=\frac{1}{\sqrt{4 \pi t}} \exp \left(-\frac{t}{4}-\frac{u^{2}}{4 t}\right)$ in Proposition 2.7, then we have

$$
\theta_{2}(t)-1=I_{2}(t)+E_{2}(t)+H E_{2}(t)+P S_{2}(t)+H S_{2}(t) .
$$

Here,

- $I_{2}(t)=\frac{\operatorname{vol}\left(\Gamma_{K} \backslash \mathbb{H}^{2}\right)}{16 \pi^{2}} \int_{-\infty}^{\infty} \exp \left(t\left(r^{2}+1 / 4\right)\right) r \tanh (\pi r) d r$

- $E_{2}(t)=-\sum_{R\left(\theta_{1}, \theta_{2}\right) \in \Gamma_{\mathrm{E}}} \frac{i e^{-i \theta_{1}}}{8 \nu_{R} \sin \theta_{1}}$

$$
\times \int_{-\infty}^{\infty} \frac{1}{\sqrt{4 \pi t}} \exp \left(-\frac{t}{4}-\frac{u^{2}}{4 t}\right) e^{-u / 2}\left[\frac{e^{u}-e^{2 i \theta_{1}}}{\cosh u-\cos 2 \theta_{1}}\right] d u
$$

- $H E_{2}(t)=-\frac{1}{2} \sum_{(\gamma, \omega) \in \Gamma_{\mathrm{HE}}} \frac{\log N\left(\gamma_{0}\right)}{N(\gamma)^{1 / 2}-N(\gamma)^{-1 / 2}}$

$$
\times \frac{1}{\sqrt{4 \pi t}} \exp \left(-\frac{t}{4}-\frac{(\log N(\gamma))^{2}}{4 t}\right),
$$

- $P S_{2}(t)=-\log \varepsilon \frac{1}{\sqrt{4 \pi t}} \exp \left(-\frac{t}{4}\right)$,

- $H S_{2}(t)=-2 \log \varepsilon \sum_{k=1}^{\infty} \frac{1}{\sqrt{4 \pi t}} \exp \left(-\frac{t}{4}-\frac{(2 k \log \varepsilon)^{2}}{4 t}\right) \varepsilon^{-k}$. 
Firstly, we see that $H E_{2}(t)$ and $H S_{2}(t)$ are exponentially decreasing as $t \rightarrow+0$. Secondly, by changing the variable $u$ to $\sqrt{t} u$ in $E_{2}(t)$, we see that there is a constant $b_{0}(2)$ such that $E_{2}(t)=b_{0}(2)+o(1)(t \rightarrow+0)$. Thirdly, $P S_{2}(t)=-\log \varepsilon \frac{1}{\sqrt{4 \pi t}}(1-t / 4+o(t))(t \rightarrow+0)$. Lastly, noting $\frac{\operatorname{vol}\left(\Gamma_{K} \backslash \mathbb{H}^{2}\right)}{8 \pi^{2}}=\zeta_{K}(-1)$ and integration by parts, we have

$$
\begin{aligned}
I_{2}(t) & =\frac{1}{2} \zeta_{K}(-1) \frac{1}{2 t} \int_{-\infty}^{\infty} \exp \left(-t\left(r^{2}+\frac{1}{4}\right)\right) \frac{\pi}{\cosh ^{2}(\pi r)} d r \\
& =\frac{\pi}{4} \zeta_{K}(-1) \sum_{n=0}^{\infty} \frac{(-1)^{n} t^{n-1}}{n !} \int_{-\infty}^{\infty} \frac{\left(r^{2}+\frac{1}{4}\right)^{n}}{\cosh ^{2}(\pi r)} d r \\
& =\frac{a_{-1}(2)}{t}+a_{0}(2)+o(1) \quad(t \rightarrow+0) .
\end{aligned}
$$

We calculate the coefficients $a_{n}(2)(n=-1,0)$.

$$
\begin{aligned}
a_{-1}(2) & =\frac{\pi}{4} \zeta_{K}(-1) \int_{-\infty}^{\infty} \frac{d r}{\cosh ^{2}(\pi r)} \\
& =\frac{\pi}{4} \zeta_{K}(-1) \frac{4}{\pi} \int_{0}^{\infty} \frac{x}{\left(x^{2}+1\right)^{2}} d x=\frac{1}{2} \zeta_{K}(-1), \\
a_{0}(2) & =-\frac{\pi}{4} \zeta_{K}(-1)\left\{\int_{-\infty}^{\infty} \frac{r^{2}}{\cosh ^{2}(\pi r)} d r+\frac{1}{4} \int_{-\infty}^{\infty} \frac{d r}{\cosh ^{2}(\pi r)}\right\} \\
& =-\frac{\pi}{4} \zeta_{K}(-1)\left(\frac{1}{6 \pi}+\frac{1}{4} \frac{2}{\pi}\right)=-\frac{1}{6} \zeta_{K}(-1) .
\end{aligned}
$$

Here, we used the formula

$$
\int_{0}^{\infty} \frac{r^{2}}{\cosh ^{2}(\pi r)} d r=\frac{\left(2^{2}-2\right) \pi^{2}}{(2 \pi)^{2} \pi} \frac{1}{6}=\frac{1}{12 \pi}
$$

in $\left[8,3.527\right.$ no. 5]. Besides, we calculate the coefficient $b_{0}(2)$ appearing in $E_{2}(t)$.

$$
\begin{aligned}
b_{0}(2) & =-\sum_{R\left(\theta_{1}, \theta_{2}\right) \in \Gamma_{\mathrm{E}}} \frac{i e^{-i \theta_{1}}}{8 \nu_{R} \sin \theta_{1}} \int_{-\infty}^{\infty} \frac{1}{\sqrt{4 \pi}} \exp \left(-\frac{u^{2}}{4}\right)\left[\frac{1-e^{2 i \theta_{1}}}{1-\cos 2 \theta_{1}}\right] d u \\
& =-\sum_{j=1}^{N} \sum_{k=1}^{\nu_{j}-1} \frac{1}{4 \nu_{j}} \frac{1}{1-\cos \left(\frac{2 \pi k}{\nu_{j}}\right)}=-\sum_{j=1}^{N} \frac{\nu_{j}^{2}-1}{24 \nu_{j}} .
\end{aligned}
$$

Summing up each terms appearing in the right hand side of (4.4), we have the desired formula (4.2). 
Let us prove (4.3) with $m=4$. For $t>0$, we also take the pair of test functions $h_{1}(r)=e^{-t\left(r^{2}+1 / 4\right)}$ and $g_{1}(u)=\frac{1}{\sqrt{4 \pi t}} \exp \left(-\frac{t}{4}-\frac{u^{2}}{4 t}\right)$ in Proposition 2.8 with $m=4$, then we have

$$
\theta_{4}(t)-\theta_{2}(t)+1=I_{4}(t)+E_{4}(t)+H E_{4}(t)+H S_{4}(t) .
$$

Here,

$$
\begin{aligned}
\text { - } I_{4}(t)= & \frac{\operatorname{vol}\left(\Gamma_{K} \backslash \mathbb{H}^{2}\right)}{8 \pi^{2}} \int_{-\infty}^{\infty} \exp \left(-t\left(r^{2}+1 / 4\right)\right) r \tanh (\pi r) d r \\
\text { - } E_{4}(t)=- & \sum_{R\left(\theta_{1}, \theta_{2}\right) \in \Gamma_{\mathrm{E}}} \frac{i e^{-i \theta_{1}} e^{2 i \theta_{2}}}{4 \nu_{R} \sin \theta_{1}} \\
& \times \int_{-\infty}^{\infty} \frac{1}{\sqrt{4 \pi t}} \exp \left(-\frac{t}{4}-\frac{u^{2}}{4 t}\right) e^{-u / 2}\left[\frac{e^{u}-e^{2 i \theta_{1}}}{\cosh u-\cos 2 \theta_{1}}\right] d u
\end{aligned}
$$

- $H E_{4}(t)=-\sum_{(\gamma, \omega) \in \Gamma_{\mathrm{HE}}} \frac{\log N\left(\gamma_{0}\right)}{N(\gamma)^{1 / 2}-N(\gamma)^{-1 / 2}}$

$$
\times \frac{1}{\sqrt{4 \pi t}} \exp \left(-\frac{t}{4}-\frac{(\log N(\gamma))^{2}}{4 t}\right) e^{2 i \omega},
$$

- $H S_{4}(t)=-2 \log \varepsilon \sum_{k=1}^{\infty} \frac{1}{\sqrt{4 \pi t}} \exp \left(-\frac{t}{4}-\frac{(2 k \log \varepsilon)^{2}}{4 t}\right)\left(\varepsilon^{-3 k}-\varepsilon^{-k}\right)$.

Similarly, we see that $H E_{4}(t)$ and $H S_{4}(t)$ are exponentially decreasing as $t \rightarrow+0$, and there is a constant $b_{0}(4)$ such that $E_{4}(t)=b_{0}(4)+o(1)$ $(t \rightarrow+0)$, and $I_{4}(t)=\zeta_{K}(-1)(1 / t-1 / 3)+o(1)(t \rightarrow+0)$. Summing up each terms appearing in the right hand side of (4.5) and using (4.2) in the left side, we have the desired formula (4.3) with $m=4$. One can prove (4.3) for $m \geq 6$ similarly. We complete the proof.

Proposition 4.3. Let $s$ be a fixed sufficiently large real number. For $m \in 2 \mathbb{N}$, let

$$
\zeta_{m}(w, s):=\sum_{n=0}^{\infty} \frac{1}{\left(\lambda_{n}(m)+s(s-1)\right)^{w}} \quad(\operatorname{Re}(w) \gg 0)
$$

be the spectral zeta function for $\square_{m}$. Then $\zeta_{m}(w, s)$ is holomorphic at $w=$ 0 .

Proof: We follow [2, p. 448]. For $w \in \mathbb{C}$ with $\operatorname{Re}(w) \gg 0$, we have

$$
\zeta_{m}(w, s)=\frac{1}{\Gamma(w)} \int_{0}^{\infty} \theta_{m}(t) e^{-s(s-1) t} t^{w} \frac{d t}{t} .
$$


We consider the first three terms of $\theta_{m}(t)$ in Proposition 4.2. Let

$$
\begin{aligned}
\eta_{p}(w, s) & :=\frac{1}{\Gamma(w)} \int_{0}^{\infty} t^{-p} e^{-s(s-1) t} t^{w-1} d t \\
& =\frac{1}{\Gamma(w)}(s(s-1))^{p-w} \Gamma(w-p)
\end{aligned}
$$

with $p=0, \frac{1}{2}, 1$. Then we see that $\eta_{p}(w, s)\left(p=0, \frac{1}{2}, 1\right)$ are holomorphic at $w=0$. The reminder term is

$$
\eta_{f}(w, s):=\frac{1}{\Gamma(w)} \int_{0}^{\infty} f(t) e^{-s(s-1) t} t^{w} \frac{d t}{t}
$$

with $f(t)=o(1)(t \rightarrow+0)$ and $O(1)(t \rightarrow \infty)$. Since $\frac{1}{\Gamma(w)}$ vanishes at $w=0$, it completes the proof.

Proposition 4.4. Let $m$ be an even natural number. We have

$$
\begin{aligned}
-\left.\frac{\partial}{\partial w} \zeta_{2}(w, s)\right|_{w=0}= & -\zeta_{K}(-1)\left(s^{2}-s+\frac{1}{3}\right) \log s+\frac{1}{2} \zeta_{K}(-1) s^{2} \\
& -s \log \varepsilon+\left(2 b_{0}(2)+2\right) \log s-\frac{1}{4} \zeta_{K}(-1) \\
& +\frac{1}{2} \log \varepsilon+o(1) \quad(s \rightarrow \infty),
\end{aligned}
$$

and for $m \geq 4$,

$$
\begin{aligned}
-\left.\frac{\partial}{\partial w} \zeta_{m}(w, s)\right|_{w=0}= & -(m-1) \zeta_{K}(-1)\left(s^{2}-s+\frac{1}{3}\right) \log s \\
& +\frac{m-1}{2} \zeta_{K}(-1) s^{2}-s \log \varepsilon \\
& +\left(2 b_{0}(2)+\cdots+2 b_{0}(m)\right) \log s \\
& -\frac{m-1}{4} \zeta_{K}(-1)+\frac{1}{2} \log \varepsilon+o(1) \quad(s \rightarrow \infty) .
\end{aligned}
$$


Besides, we have for $m \geq 4$,

$$
\begin{aligned}
-\left.\frac{\partial}{\partial w} \zeta_{m}(w, s)\right|_{w=0} & +\left.\frac{\partial}{\partial w} \zeta_{m-2}(w, s)\right|_{w=0} \\
= & -2 \zeta_{K}(-1)\left(s^{2}-s+\frac{1}{3}\right) \log s \\
& +\zeta_{K}(-1) s^{2}+\left(2 b_{0}(m)-2 \delta_{4, m}\right) \log s \\
& -\frac{1}{2} \zeta_{K}(-1)+o(1) \quad(s \rightarrow \infty) .
\end{aligned}
$$

Proof: By the formulas (4.7) and (4.8), we find that

$$
\begin{array}{rlrl}
\left.\frac{\partial}{\partial w} \eta_{0}(w, s)\right|_{w=0} & =-\log (s(s-1))=-2 \log s+o(1) & & (s \rightarrow \infty), \\
\left.\frac{\partial}{\partial w} \eta_{\frac{1}{2}}(w, s)\right|_{w=0} & =-2 \sqrt{\pi}(s(s-1))^{\frac{1}{2}}=-2 \sqrt{\pi}\left(s-\frac{1}{2}\right)+o(1) & (s \rightarrow \infty), \\
\left.\frac{\partial}{\partial w} \eta_{1}(w, s)\right|_{w=0} & =s(s-1)(\log (s(s-1))-1) & & (s \rightarrow \infty), \\
& =2 s(s-1) \log s+\frac{1}{2}-s^{2}+o(1) & (s \rightarrow \infty) . \\
\left.\frac{\partial}{\partial w} \eta_{f}(w, s)\right|_{w=0} & =o(1) &
\end{array}
$$

Therefore, by using (4.2), we have

$$
\begin{aligned}
-\left.\frac{\partial}{\partial w} \zeta_{2}(w, s)\right|_{w=0}= & -\frac{1}{2} \zeta_{K}(-1)\left(2 s(s-1) \log s+\frac{1}{2}-s^{2}\right)-\left(s-\frac{1}{2}\right) \log \varepsilon \\
& +\left(-\frac{1}{6} \zeta_{K}(-1)+b_{0}(2)+1\right) 2 \log s+o(1) \quad(s \rightarrow \infty) \\
= & -\zeta_{K}(-1)\left(s^{2}-s+\frac{1}{3}\right) \log s+\frac{1}{2} \zeta_{K}(-1) s^{2} \\
& -s \log \varepsilon+\left(2 b_{0}(2)+2\right) \log s-\frac{1}{4} \zeta_{K}(-1) \\
& +\frac{1}{2} \log \varepsilon+o(1) \quad(s \rightarrow \infty) .
\end{aligned}
$$


For $m \geq 4$, by using (4.3), we have

$$
\begin{aligned}
-\left.\frac{\partial}{\partial w} \zeta_{m}(w, s)\right|_{w=0}= & -(m-1) \zeta_{K}(-1)\left(s^{2}-s+\frac{1}{3}\right) \log s \\
& +\frac{m-1}{2} \zeta_{K}(-1) s^{2}-s \log \varepsilon \\
& +\left(2 b_{0}(2)+\cdots+2 b_{0}(m)\right) \log s \\
& -\frac{m-1}{4} \zeta_{K}(-1)+\frac{1}{2} \log \varepsilon+o(1) \quad(s \rightarrow \infty) .
\end{aligned}
$$

We complete the proof.

\section{Proof of Main Theorem}

In this section we prove Theorem 1.6. We prove the following two propositions. The first proposition connect the completed Selberg zeta functions:

$$
\widehat{Z}_{2}^{\frac{1}{2}}(s), \widehat{Z}_{4}(s), \ldots, \widehat{Z}_{m}(s)
$$

with the regularized determinants of Laplacians:

$$
\operatorname{Det}\left(\square_{2}+s(s-1)\right), \operatorname{Det}\left(\square_{4}+s(s-1)\right), \ldots, \operatorname{Det}\left(\square_{m}+s(s-1)\right) \text {. }
$$

The second proposition determines the explicit relations among them. Theorem 1.6 is deduced from these two propositions.

Proposition 5.1. Let $\square_{m}:=\left.\Delta_{0}^{(1)}\right|_{V_{m}^{(2)}}$ for $m \in 2 \mathbb{N}$. There exit polynomials $P_{2}(s), \ldots, P_{m}(s)$ such that

$$
\begin{gathered}
\widehat{Z}_{2}^{\frac{1}{2}}(s)=e^{P_{2}(s)} \frac{\operatorname{Det}\left(\square_{2}+s(s-1)\right)}{s(s-1)}, \\
\widehat{Z}_{4}(s)=e^{P_{4}(s)} \frac{s(s-1) \operatorname{Det}\left(\square_{4}+s(s-1)\right)}{\operatorname{Det}\left(\square_{2}+s(s-1)\right)}, \\
\widehat{Z}_{m}(s)=e^{P_{m}(s)} \frac{\operatorname{Det}\left(\square_{m}+s(s-1)\right)}{\operatorname{Det}\left(\square_{m-2}+s(s-1)\right)} \quad(m \geq 6) .
\end{gathered}
$$

Proof: Let $k$ be a sufficiently large natural number. We note that

$$
\left(-\frac{1}{2 s-1} \frac{d}{d s}\right)^{k+1} \zeta_{m}(w, s)=w(w+1) \cdots(w+k) \zeta_{m}(w+k+1, s) .
$$

Taking $-\left.\frac{\partial}{\partial w}\right|_{w=0}$ of both sides, we have

$$
\left(-\frac{1}{2 s-1} \frac{d}{d s}\right)^{k+1} \log \operatorname{Det}\left(\square_{m}+s(s-1)\right)=-\sum_{j=0}^{\infty} \frac{k !}{\left(\lambda_{j}(m)+s(s-1)\right)^{k+1}}
$$


Let $m=2$, we use the following double differences of STF with the certain test function (see [6, Theorem 6.4]):

$$
\begin{aligned}
\sum_{j=0}^{\infty} & {\left[\frac{1}{\rho_{j}(2)^{2}+\left(s-\frac{1}{2}\right)^{2}}+\sum_{h=1}^{2} \frac{c_{h}(s)}{\rho_{j}(2)^{2}+\beta_{h}^{2}}\right]-\left[\frac{1}{s(s-1)}+\sum_{h=1}^{2} \frac{c_{h}(s)}{\beta_{h}^{2}-\frac{1}{4}}\right] } \\
= & \zeta_{K}(-1) \sum_{k=0}^{\infty}\left[\frac{1}{s+k}+\sum_{h=1}^{2} \frac{c_{h}(s)}{\beta_{h}+\frac{1}{2}+k}\right]+\frac{1}{2 s-1} \frac{\frac{d}{d s} \sqrt{Z_{2}(s)}}{\sqrt{Z_{2}(s)}} \\
& +\sum_{h=1}^{2} \frac{c_{h}(s)}{2 \beta_{h}} \frac{\frac{d}{d \beta_{h}} \sqrt{Z_{2}\left(\frac{1}{2}+\beta_{h}\right)}}{\sqrt{Z_{2}\left(\frac{1}{2}+\beta_{h}\right)}}+\frac{1}{2 s-1} \sum_{j=1}^{N} \sum_{l=0}^{\nu_{j}-1} \frac{\nu_{j}-1-2 l}{2 \nu_{j}^{2}} \psi\left(\frac{s+l}{\nu_{j}}\right) \\
& +\sum_{h=1}^{2} \frac{c_{h}(s)}{2 \beta_{h}} \sum_{j=1}^{N} \sum_{l=0}^{\nu_{j}-1} \frac{\nu_{j}-1-2 l}{2 \nu_{j}^{2}} \psi\left(\frac{\frac{1}{2}+\beta_{h}+l}{\nu_{j}}\right)+\frac{1}{2 s-1} \frac{d}{d s} \log \left(\varepsilon^{-s}\right) \\
& +\sum_{h=1}^{2} \frac{c_{h}(s)}{2 \beta_{h}} \frac{d}{d \beta_{h}} \log \left(\varepsilon^{-\left(\beta_{h}+1 / 2\right)}\right)+\frac{1}{2 s-1} \frac{d}{d s} \log \left\{\frac{1}{\left(1-\varepsilon^{-2 s}\right)}\right\} \\
& +\sum_{h=1}^{2} \frac{c_{h}(s)}{2 \beta_{h}} \frac{d}{d \beta_{h}} \log \left\{\frac{1}{\left(1-\varepsilon^{-\left(2 \beta_{h}+1\right)}\right)}\right\} .
\end{aligned}
$$

Here, $\psi(z)$ is the digamma function, $\beta_{1} \neq \beta_{2}$ are constants and $c_{1}(s), c_{2}(s)$ are quadratic polynomials invariant under $s \rightarrow 1-s$. Operating $\left(-\frac{1}{2 s-1} \frac{d}{d s}\right)^{k}$ on both sides, we have

$$
\begin{aligned}
& \sum_{j=0}^{\infty} \frac{k !}{\left(\lambda_{j}(2)+s(s-1)\right)^{k+1}} \\
& \quad=\left(-\frac{1}{2 s-1} \frac{d}{d s}\right)^{k} \frac{1}{2 s-1} \frac{d}{d s} \log \left(\widehat{Z}_{2}^{\frac{1}{2}}(s) s(s-1)\right) .
\end{aligned}
$$

By (5.1) and (5.2), we have

$$
\begin{aligned}
\left(-\frac{1}{2 s-1} \frac{d}{d s}\right)^{k+1} \log \operatorname{Det} & \left(\square_{2}+s(s-1)\right) \\
& =\left(-\frac{1}{2 s-1} \frac{d}{d s}\right)^{k+1} \log \left(\widehat{Z}_{2}^{\frac{1}{2}}(s) s(s-1)\right) .
\end{aligned}
$$

Therefore, we find that there exists a polynomial $P_{2}(s)$ such that

$$
\log \operatorname{Det}\left(\square_{2}+s(s-1)\right)+P_{2}(s)=\log \left(\widehat{Z}_{2}^{\frac{1}{2}}(s) s(s-1)\right) .
$$


Thus we have

$$
\widehat{Z}_{2}^{\frac{1}{2}}(s)=e^{P_{2}(s)} \frac{\operatorname{Det}\left(\square_{2}+s(s-1)\right)}{s(s-1)} .
$$

Let $m \geq 4$ be an even integer. We use the following double differences of STF with the certain test function (see [6, Theorem 5.2]):

$$
\begin{aligned}
& \sum_{j=0}^{\infty}\left[\frac{1}{\rho_{j}(m)^{2}+\left(s-\frac{1}{2}\right)^{2}}+\sum_{h=1}^{2} \frac{c_{h}(s)}{\rho_{j}(m)^{2}+\beta_{h}^{2}}\right] \\
& -\sum_{j=0}^{\infty}\left[\frac{1}{\rho_{j}(m-2)^{2}+\left(s-\frac{1}{2}\right)^{2}}+\sum_{h=1}^{2} \frac{c_{h}(s)}{\rho_{j}(m-2)^{2}+\beta_{h}^{2}}\right] \\
& +\delta_{m, 4}\left[\frac{1}{s(s-1)}+\sum_{h=1}^{2} \frac{c_{h}(s)}{\beta_{h}^{2}-\frac{1}{4}}\right] \\
& =2 \zeta_{K}(-1) \sum_{k=0}^{\infty}\left[\frac{1}{s+k}+\sum_{h=1}^{2} \frac{c_{h}(s)}{\beta_{h}+\frac{1}{2}+k}\right] \\
& +\frac{1}{2 s-1} \frac{Z_{m}^{\prime}(s)}{Z_{m}(s)}+\sum_{h=1}^{2} \frac{c_{h}(s)}{2 \beta_{h}} \frac{Z_{m}^{\prime}\left(\frac{1}{2}+\beta_{h}\right)}{Z_{m}\left(\frac{1}{2}+\beta_{h}\right)} \\
& +\frac{1}{2 s-1} \sum_{j=1}^{N} \sum_{l=0}^{\nu_{j}-1} \frac{\nu_{j}-1-\alpha_{l}(m, j)-\overline{\alpha_{l}}(m, j)}{\nu_{j}^{2}} \psi\left(\frac{s+l}{\nu_{j}}\right) \\
& +\sum_{h=1}^{2} \frac{c_{h}(s)}{2 \beta_{h}} \sum_{j=1}^{N} \sum_{l=0}^{\nu_{j}-1} \frac{\nu_{j}-1-\alpha_{l}(m, j)-\overline{\alpha_{l}}(m, j)}{\nu_{j}^{2}} \psi\left(\frac{\frac{1}{2}+\beta_{h}+l}{\nu_{j}}\right) \\
& +\frac{1}{2 s-1} \frac{d}{d s} \log \left\{\frac{\left(1-\varepsilon^{-(2 s+m-4)}\right)}{\left(1-\varepsilon^{-(2 s+m-2)}\right)}\right\} \\
& +\sum_{h=1}^{2} \frac{c_{h}(s)}{2 \beta_{h}} \frac{d}{d \beta_{h}} \log \left\{\frac{\left(1-\varepsilon^{-\left(2 \beta_{h}+m-3\right)}\right)}{\left(1-\varepsilon^{-\left(2 \beta_{h}+m-1\right)}\right)}\right\} .
\end{aligned}
$$

Here, $\beta_{1} \neq \beta_{2}$ are constants and $c_{1}(s), c_{2}(s)$ are quadratic polynomials invariant under $s \rightarrow 1-s$. 
Operating $\left(-\frac{1}{2 s-1} \frac{d}{d s}\right)^{k}$ on both sides, we have

$$
\begin{aligned}
& \sum_{j=0}^{\infty} \frac{k !}{\left(\lambda_{j}(m)+s(s-1)\right)^{k+1}}-\sum_{j=0}^{\infty} \frac{k !}{\left(\lambda_{j}(m-2)+s(s-1)\right)^{k+1}} \\
& \quad=\left(-\frac{1}{2 s-1} \frac{d}{d s}\right)^{k} \frac{1}{2 s-1} \frac{d}{d s}\left(\log \widehat{Z}_{m}(s)-\delta_{m, 4} \log (s(s-1))\right) .
\end{aligned}
$$

By (5.1) and (5.4), there exists a polynomial $P_{m}(s)$ such that

(5.5) $\log \operatorname{Det}\left(\square_{m}+s(s-1)\right)-\log \operatorname{Det}\left(\square_{m-2}+s(s-1)\right)+P_{m}(s)$

$$
=\log \widehat{Z}_{m}(s)-\delta_{m, 4} \log (s(s-1)) \text {. }
$$

We complete the proof.

Proposition 5.2. We have

$$
\begin{aligned}
& P_{2}(s)=\left(s-\frac{1}{2}\right)^{2} \zeta_{K}(-1)-\frac{1}{2} \log \varepsilon+\sum_{j=1}^{N} \frac{\nu_{j}^{2}-1}{12 \nu_{j}} \log \nu_{j}, \\
& P_{m}(s)=2\left(s-\frac{1}{2}\right)^{2} \zeta_{K}(-1)+\sum_{j=1}^{N} \frac{\nu_{j}^{2}-1-12 \alpha_{0}(m, j)\left\{\nu_{j}-\alpha_{0}(m, j)\right\}}{6 \nu_{j}} \log \nu_{j}
\end{aligned}
$$

Proof: Substituting (3.6) and (4.9) in (5.3), we have

$$
\begin{aligned}
P_{2}(s)= & \log \left(\widehat{Z}_{2}^{\frac{1}{2}}(s) s(s-1)\right)-\log \operatorname{Det}\left(\square_{2}+s(s-1)\right) \\
= & \zeta_{K}(-1)\left\{\frac{3}{2} s^{2}-s-\left(s^{2}-s+\frac{1}{3}\right) \log s\right\}-\sum_{j=1}^{N} \frac{\nu_{j}^{2}-1}{12 \nu_{j}} \log \frac{s}{\nu_{j}} \\
& -s \log \varepsilon+2 \log s+\zeta_{K}(-1)\left(s^{2}-s+\frac{1}{3}\right) \log s-\frac{1}{2} \zeta_{K}(-1) s^{2} \\
& +s \log \varepsilon-\left(2 b_{0}(2)+2\right) \log s+\frac{1}{4} \zeta_{K}(-1)-\frac{1}{2} \log \varepsilon+o(1) \\
= & \left(s-\frac{1}{2}\right)^{2} \zeta_{K}(-1)-\frac{1}{2} \log \varepsilon+\sum_{j=1}^{N} \frac{\nu_{j}^{2}-1}{12 \nu_{j}} \log \nu_{j}+o(1) \quad(s \rightarrow \infty) .
\end{aligned}
$$

Since $P_{2}(s)$ is a polynomial, we have the desired formula for $P_{2}(s)$. 
Let $m \geq 4$. Substituting (3.7) and (4.11) in (5.5), we have

$$
\begin{aligned}
P_{m}(s)= & \log \widehat{Z}_{m}(s)-\delta_{m, 4} \log (s(s-1)) \\
& -\log \operatorname{Det}\left(\square_{m}+s(s-1)\right)+\log \operatorname{Det}\left(\square_{m-2}+s(s-1)\right) \\
= & 2\left(s-\frac{1}{2}\right)^{2} \zeta_{K}(-1) \\
& +\sum_{j=1}^{N} \frac{\nu_{j}^{2}-1-12 \alpha_{0}(m, j)\left\{\nu_{j}-\alpha_{0}(m, j)\right\}}{6 \nu_{j}} \log \nu_{j}+o(1) \quad(s \rightarrow \infty) .
\end{aligned}
$$

Since $P_{m}(s)$ is a polynomial, we have the desired formula for $P_{m}(s)$. It completes the proof.

\section{Acknowledgement}

The author would like to thank the referee for careful reading of the first version and many helpful suggestions for the revision of this paper.

\section{References}

[1] E. W. Barnes, The theory of the G-function, Quart. J. 31 (1900), 264-314.

[2] I. EFRAT, Determinants of Laplacians on surfaces of finite volume, Comm. Math. Phys. 119(3) (1988), 443-451; Erratum: "Determinants of Laplacians on surfaces of finite volume", Comm. Math. Phys. 138(3) (1991), 607.

[3] G. Freixas i Montplet, An arithmetic Riemann-Roch theorem for pointed stable curves, Ann. Sci. Éc. Norm. Supér. (4) 42(2) (2009), 335-369. DOI: 10.24033/asens. 2098.

[4] G. Freixas i Montplet and A. von Pippich, Riemann-Roch isometries in the non-compact orbifold setting, Preprint (2016). arXiv: 1604.00284.

[5] Y. Gon, Selberg type zeta function for the Hilbert modular group of a real quadratic field, Proc. Japan Acad. Ser. A Math. Sci. 88(9) (2012), 145-148. DOI: 10.3792/pjaa.88.145.

[6] Y. Gon, Differences of the Selberg trace formula and Selberg type zeta functions for Hilbert modular surfaces, J. Number Theory 147 (2015), 396-453. DOI: 10.1016/j.jnt.2014.07.019.

[7] Y. GON AND J. PARK, The zeta functions of Ruelle and Selberg for hyperbolic manifolds with cusps, Math. Ann. 346(3) (2010), 719-767. DOI : 10.1007/s00208-009-0408-7. 
[8] I. S. Gradshteyn And I. M. Ryzhik, "Table of Integrals, Series, and Products", Translated from the Russian, Translation edited and with a preface by Alan Jeffrey and Daniel Zwillinger, With one CD-ROM (Windows, Macintosh and UNIX), Seventh edition, Elsevier/Academic Press, Amsterdam, 2007.

[9] F. Hirzebruch And D. ZAGier, Classification of Hilbert modular surfaces, in: "Complex Analysis and Algebraic Geometry", Iwanami Shoten, Tokyo, 1977, pp. 43-77.

[10] S. Koyama, Determinant expression of Selberg zeta functions. I, Trans. Amer. Math. Soc. 324(1) (1991), 149-168. DOI: 10.2307/ 2001500.

[11] N. Kurokawa, Parabolic components of zeta functions, Proc. Japan Acad. Ser. A Math. Sci. 64(1) (1988), 21-24. DOI: 10.3792/ pjaa.64.21.

[12] N. Kurokawa and S. Koyama, Multiple sine functions, Forum Math. 15(6) (2003), 839-876. DOI: 10.1515/form.2003.042.

[13] N. N. Lebedev, "Special Functions and their Applications", Revised edition, Translated from the Russian and edited by Richard A. Silverman, Unabridged and corrected republication, Dover Publications, Inc., New York, 1972.

[14] K. OnODERA, Weierstrass product representations of multiple gamma and sine functions, Kodai Math. J. 32(1) (2009), 77-90. DOI : $10.2996 / \mathrm{kmj} / 1238594547$.

[15] P. SARnAK, Determinants of Laplacians, Comm. Math. Phys. 110(1) (1987), 113-120.

[16] A. Selberg, Harmonic analysis and discontinuous groups in weakly symmetric Riemannian spaces with applications to Dirichlet series, J. Indian Math. Soc. (N.S.) 20 (1956), 47-87.

Faculty of Mathematics

Kyushu University

744 Motooka, Nishi-ku

Fukuoka 819-0395

Japan

E-mail address: ygon@math.kyushu-u.ac.jp

Primera versió rebuda el 27 de gener de 2017, darrera versió rebuda el 18 d'abril de 2017. 